\title{
Práticas parentais coercitivas e as repercussões nos problemas de comportamento dos filhos
}

\author{
Coercive parenting practices and the impact on behavior problems of children
}

\author{
Marcela Bortolini ${ }^{[a]}$, llana Andretta ${ }^{[b]}$
}

\footnotetext{
${ }^{[a]}$ Acadêmica da Faculdade de Psicologia da Pontifícia Universidade Católica do Rio Grande do Sul (PUCRS), Porto Alegre, RS - Brasil, e-mail: bortolini.marcela@gmail.com

${ }^{[b]}$ Doutora em Psicologia pela Pontifícia Universidade Católica do Rio Grande do Sul (PUCRS), professora adjunta da Faculdade de Psicologia da Pontifícia Universidade Católica do Rio Grande do Sul (PUCRS), Porto Alegre, RS -Brasil, e-mail: ilana.andretta@gmail.com
}

Recebido: 01/02/2011 Received: 02/01/2011

Aprovado: 05/04/2011 Approved: 04/05/2011

\begin{abstract}
Resumo
Práticas educacionais parentais são estratégias utilizadas pelos pais para educar os filhos segundo as próprias considerações. Podem ser divididas em práticas parentais coercitivas e não coercitivas; as primeiras são caracterizadas pelo uso de estímulos aversivos, propiciando o desenvolvimento de problemas de comportamento, essencialmente problemas de comportamento externalizantes, e as segundas caracterizam-se pelo uso de reforçadores positivos. A associação entre práticas parentais coercitivas com problemas de externalização nas crianças é frequentemente referida na literatura. 0 objetivo do presente estudo foi analisar a relação entre o uso de práticas educacionais coercitivas e as repercussões dessas nos problemas de comportamento dos filhos, além de analisar a utilização do Treinamento de Pais como meio de reeducar a maneira dos pais de interagir com seus filhos sem o uso de métodos punitivos e os benefícios deste uso. Realizou-se uma investigação a partir de dois estudos de caso com enfoque qualitativo. Em ambos os estudos de caso verificou-se a presença de problemas de comportamentos externalizantes nos filhos que sofriam do uso de práticas parentais coercitivas. Constataram-se algumas dificuldades de adesão dos cuidadores às técnicas que contemplam o Treinamento de Pais, bem como resultados positivos na relação e nos comportamentos dos filhos com seus pais quando estes utilizaram as técnicas. Considera-se importante o uso de práticas parentais não coercitivas como meio de proteção ao desenvolvimento de crianças que futuramente estarão inseridas na sociedade com comportamentos mais humanitários a partir de aprendizagens mais saudáveis e positivas com seus pais.
\end{abstract}

Palavras-chave: Relações pai-filho. Comportamento. Agressão. 
relationship between the use of coercive educational practices and their impact on behavior problems of children, in addition to analyzing the use of the Training of Parents as a means to re-educate the way for parents to interact their children without the use of punitive methods and benefits of use. We conducted an investigation through two case studies with qualitative approach. In both case studies verified the presence of problems of externalizing behaviors in children who suffered from the use of coercive parenting practices. We also contacted some difficulties in adherence to the techniques of caregivers that include the Training of Parents as well as positive results in the relationship and behavior of children with their parents when they could use the techniques. It is considered important to use non-coercive parenting practices as a means of protecting the future development of children who are placed in society to humanitarian behaviors before learning more healthy and positive with their parents.

Keywords: Parent-child relationships. Behavior. Agression.

\section{Introdução}

As práticas educacionais parentais, segundo Salvador e Weber (2005), correspondem a estratégias utilizadas pelos pais para suprimir comportamentos considerados inadequados ou incentivar a ocorrência de comportamentos adequados na educação dos filhos. Os pais as utilizam para desenvolver disciplina e promover socialização, independência, autonomia e responsabilidade a fim de modelar os comportamentos dos filhos segundo as próprias considerações (Sapienza, Aznar-Farias \& Silvares, 2009). Essas práticas educacionais estão relacionadas com o desenvolvimento saudável da criança assim como com a presença de comportamentos antissociais (Gomide, 2001).

Práticas educacionais parentais podem ser divididas em práticas parentais não coercitivas, também chamadas de práticas positivas, e práticas parentais coercitivas, também chamadas de práticas negativas. As primeiras utilizam essencialmente reforçadores positivos e regras, e as últimas, estímulos aversivos (Salvador \& Weber, 2005). Pais com menos habilidades e com menor disponibilidade emocional para perceber os sinais de seus bebês tendem a empregar com maior frequência práticas coercitivas. Por outro lado, pais mais sensíveis ao comportamento de seus bebês tendem a ser mais hábeis na regulação do comportamento dos filhos, conseguindo utilizar estratégias assertivas e reforçadoras em etapas posteriores do desenvolvimento (Alvarenga \& Piccinini, 2007). A falta de habilidades parentais é em parte responsável pelo desenvolvimento ou manutenção de interações familiares perturbadoras e, consequentemente, problemas de comportamentos nos filhos (Coelho \& Murta, 2007). Lubi (2003) destaca que o desenvolvimento de habilidades sociais na primeira infância está relacionado ao contexto familiar, às vivências e às práticas educacionais.

Alguns autores especificam as práticas educacionais parentais em sete categorias, duas correspondendo às práticas parentais positivas e cinco às negativas. São especificadas nas práticas educacionais positivas as práticas de monitoria positiva e a de comportamento moral. A primeira consiste em demonstrações de carinho, afeto, atenção e conhecimento sobre as atividades dos filhos; a segunda refere-se ao ensinamento de valores morais, honestidade, generosidade e senso de justiça (Salvo, Silvares \& Toni, 2005). Outras condutas parentais também estão associadas ao desenvolvimento saudável, dentre elas: condução calorosa, demonstração de aceitação, valorização pessoal, apoio às iniciativas, encorajamento ao desenvolvimento de competência social, interações mais positivas, expressão contínua de afeto positivo, modelos apropriados de pais, incentivo ao desenvolvimento da autonomia, utilização de métodos racionais e verbais de disciplina e minimização de brigas e agressões entre familiares (Conte, 2001).

Dentre as práticas parentais negativas categorizadas por Salvo, Silvares e Toni (2005) são especificadas as seguintes práticas: de punição inconsistente, que acontecem quando os pais punem ou reforçam os comportamentos de seus filhos de acordo com o humor e não de modo contingente ao comportamento da criança; de negligência, que se caracteriza pelos pais não estarem atentos às 
necessidades de seus filhos, ocorrendo omissão de auxílio, afeto e amor; de monitoria negativa, que se constitui pelo excesso de fiscalização e de instruções que não são seguidas pelos filhos, dificultando, dessa maneira, a dependência emocional das crianças aos pais; de disciplina relaxada, a qual ocorre quando os pais não cumprem as regras estabelecidas e assim a criança pode tornar-se rebelde e manipuladora; e de abuso físico, que acontece quando os pais machucam ou causam dor a seus filhos com a justificativa de que estão educando. Destaca-se que o abuso físico e a negligência são os fatores que desencadeiam com maior facilidade comportamentos antissociais em crianças e adolescentes.

A interação dos cuidadores com os filhos nos anos iniciais tem sido considerada a base para o sucesso das relações futuras (Piccinini, Frizzo, Alvarenga, Lopes \& Tudge, 2007); isso porque experiências vivenciadas pelas crianças nos primeiros anos repercutem nos comportamentos ao longo do desenvolvimento, culminando com o surgimento ou não de problemas de comportamento na infância média e na adolescência (Bandeira, Rocha, Souza, Del Prette Z. \& Del Prette A., 2006). Crianças que acumulam uma variedade de problemas de comportamento ao longo da trajetória correm o risco de desenvolver uma vasta gama de resultados negativos, como abandono escolar, parentalidade na adolescência, delinquência e violência (Lochman, Powell, Whidby \& Fitzgerald, 2006).

A associação entre práticas parentais coercitivas com problemas de externalização nas crianças é frequentemente referida na literatura (Alvarenga \& Piccinini, 2007; Pesce, 2009; Piccinini et al., 2007). Problemas externalizantes envolvem a presença de agressividade física e/ou verbal, comportamentos opositores ou desafiantes, condutas antissociais, assim como comportamentos de risco (Bandeira et al., 2006).

Del Prette, A. e Del Prette, Z. (2002) referem três modos utilizados pelos pais para educar os filhos: por meio da modelagem (Teoria da Aprendizagem Social), por meio de consequências (recompensas e punições) e pelo estabelecimento de normas, explicações, exortações e estímulos. Bandura (1973) propõe, por meio da Teoria Social da Aprendizagem, que crianças podem aprender comportamentos complexos perante a observação de um modelo (pais/responsáveis) conquistando uma aprendizagem muito resistente à extinção. Essa teoria é extremamente importante para a compreensão da agressividade humana, uma vez que focaliza o papel do ambiente social na aquisição, manutenção e modificação das respostas agressivas (Cortez, Padovani \& Williams, 2005). Essa relação é percebida em famílias com crianças agressivas, nas quais tanto os pais quanto os filhos utilizam respostas aversivas para lidar com conflitos (Gallo \& Williams, 2005). Compreende-se que pais que utilizam punição verbal, psicológica ou física mostram aos filhos que a violência é uma maneira apropriada para se relacionar e de resolver conflitos (Pesce, 2009).

Conforme Bandura (1973), os valores e as condutas agressivas dos adultos servem como normas a serem seguidas pelos filhos, e os mesmos tendem a imitar essas práticas parentais. A aprendizagem da agressividade por meio de modelos (família, sociedade, ídolos) acontece quando o individuo está atento às dicas e pistas que lhe são dadas, às observações que são codificadas e representadas na memória, às representações transformadas em padrões de imitação de comportamento e aos incentivos à atuação do que foi aprendido. Esses incentivos são aprendidos lentamente pelas recompensas e punições que os modelos mostram (Kristensen, Lima, Ferlin, Flores \& Hackmann, 2003). Logo, muitos desvios às normas podem ser explicados pelos tipos de informação a que o indivíduo tem acesso e pela importância dada a essas informações (Gallo \& Williams, 2005).

Comportamentos com consequências fortalecedoras e sentimentos positivos são valorados como bons, são reforços; já comportamentos com consequências enfraquecedoras e sentimentos negativos são valorados como maus, são punições. As consequências reforçadoras fortalecem o comportamento, ao passo que as punitivas enfraquecem o comportamento. Assim, é com base no efeito das consequências do comportamento e nos sentimentos que o acompanham que os indivíduos formulam juízos de valor ou atribuem valor às coisas (Abib, 2001). As influências exercidas pelos comportamentos de um modelo (pais) no comportamento de um indivíduo (filho) envolvem a generalização dos padrões de resposta imitativa em novos contextos em que o modelo pode estar ausente (Marlowe \& Canestrari, 2006). Corroborando com a ocorrência dessa generalização, Bolsoni-Silva e Marturano (2002) descrevem que os comportamentos perturbadores de crianças tendem a ocorrer com o passar do tempo em diferentes locais, passando, por 
exemplo, da casa ou pré-escola para locais escolares e depois para comunidades mais amplas.

Considerando os reforços e punições, entende-se que pais e filhos treinam uns aos outros. A probabilidade de a criança desenvolver comportamentos agressivos e desafiadores pode vir a aumentar quando as crianças recusam-se a seguir o comportamento exigido pelos pais e estes se deparam com a inabilidade em lidar com a recusa. Diante dessa dinâmica, o comportamento da criança pode assumir um caráter aversivo tendendo a agravar-se gradualmente e seria reforçado negativamente pela desistência dos pais, ficando mais frequente e intenso o comportamento não desejado (Todorov \& Moreira, 2009). Segundo Skinner (1971) citado por Abib, 2001, p. 28, as práticas ameaçadoras que produzem reforço positivo imediato com consequências negativas postergadas precisam ser enfrentadas com a modificação das contingências, programando-se consequências negativas mais imediatas para essas práticas ou fortalecer práticas alternativas com condições de substituí-las. Cabe ressaltar que muitas vezes os comportamentos pró-sociais das crianças são frequentemente ignorados ou respondidos de maneiras inapropriadas, não sendo reforçados ou até mesmo punidos pelos pais (Bolsoni-Silva, Del Prette \& Oishi, 2003).

Diante dos estudos referidos e do estudo recente de Velásquez, Souza, Adjuto, Muñoz e Silveira (2010), conclui-se que as práticas educativas parentais coercitivas tornam os filhos mais agressivos e como consequência essas crianças aprendem a valorizar a força e o poder nos relacionamentos, de modo a reproduzir o comportamento agressivo dos pais. Assim, o surgimento de problemas de comportamentos e emocionais em crianças e adolescentes motiva o desenvolvimento de intervenções dirigidas aos pais, como por exemplo, o Treinamento de Pais (Coelho \& Murta, 2007). Como afirma SerraPinheiro, Guimarães e Serrano (2005, p. 69),

treinamento de pais consiste em ensinar aos pais o que modula o comportamento das crianças e influência a chance dele ocorrer novamente. Além de ensinar uma série de técnicas comportamentais envolvendo uso de atenção diferenciada, sistemas de remuneração e de restrições de remuneração, além de planejamento de situações de potencial conforto.

Cuidadores que participaram do Treinamento de Pais em um estudo relataram mudanças no desenvolvimento de novas habilidades sociais dos seus filhos como qualidade positiva nas relações com seus dependentes, redução de problemas de comportamento e diminuição de práticas violentas na educação dos filhos (Velásquez et al., 2010), melhoras nas atividades acadêmicas, aumento no repertório de solução de problemas e desenvolvimento de cuidados em prol da saúde (Coelho \& Murta, 2007). Corroborando com essas mudanças positivas, no estudo de Serra-Pinheiro et al. (2005) também foi constatada uma redução dos sintomas de problemas de comportamentos nas crianças com o diagnóstico de Transtorno Desafiador de Oposição e Transtorno de Conduta, cujos pais participaram do Treinamento de Pais.

Perante as constatações das influências das práticas parentais no comportamento dos filhos, torna-se imprescindível que pais alterem suas práticas educativas para promoverem repertório socialmente adequado em seus filhos (Bolsoni-Silva et al., 2003) e para não ensinar aos seus filhos os próprios estilos e práticas parentais inadequadas, visto que é por meio dos modelos familiares que os estilos e práticas muitas vezes são aprendidos de geração para geração (Weber, Selig, Bernardi \& Salvador, 2006).

0 objetivo do presente estudo foi analisar a relação entre o uso de práticas educacionais coercitivas e as repercussões dessas nos problemas de comportamento dos filhos, além de analisar a utilização do Treinamento de Pais como meio de reeducar a maneira dos pais de educar seus filhos sem o uso de métodos punitivos e os benefícios deste uso.

\section{Metodologia}

Realizou-se uma investigação a partir de dois estudos de caso com enfoque de investigação qualitativa. Ressalta-se que o estudo de caso tem como característica estudar uma unidade bem delimitada e contextualizada, com a preocupação de não analisar apenas o caso em si, mas o que ele representa dentro do todo, além de ser uma das principais modalidades de pesquisa qualitativa em ciências humanas e sociais (Ventura, 2007). Geralmente é escolha para quando o pesquisador tem pouco controle sobre os acontecimentos e quando o foco se encontra em fenômenos contemporâneos inseridos em contexto de vida real (Yin, 2005).

0 presente estudo foi realizado em uma clínica-escola localizada em Porto Alegre, Rio Grande do Sul. 
O estudo contempla a compreensão de dois atendimentos conduzidos por uma estagiária do oitavo semestre da faculdade de psicologia sob supervisão semanal individual dos atendimentos realizados. 0 primeiro estudo apresenta o caso de uma criança que, inicialmente, recebera atendimento grupal e, posteriormente, individual com foco nos problemas de comportamento externalizantes, enquanto os pais realizavam o Treinamento de Pais. 0 segundo apresenta o caso de uma mulher que recebera atendimento individual para extinguir as suas práticas parentais coercitivas que estavam repercutindo na presença de comportamentos inadequados na filha. Para assegurar o sigilo, foram utilizados nomes fictícios. Além disso, os pacientes estavam cientes de pesquisas desenvolvidas na instituição ao assinaram o Termo de Consentimento Livre e Esclarecido (TCLE).

\section{Resultados}

\section{Dados pessoais e processo de atendimento psicológico}

O primeiro caso clínico aborda o processo de atendimento de Lucas (8 anos) que chega ao atendimento com queixas de desobediência aos pais, comportamentos agressivos com a mãe, a irmã e os colegas de aula, dificuldades e faltas escolares, mentiras frequentes, além de comportamentos opositores. Lucas reside com os pais e com a irmã de 5 anos em uma comunidade de baixo nível socioeconômico, o que caracteriza as dificuldades financeiras da família.

No processo de atendimento psicológico, inicialmente Lucas participou de uma intervenção grupal direcionada para crianças com problemas de comportamentos externalizantes; ele mentia para os pais, agredia outros meninos, fugia de casa, desobedecia às ordens dos pais, não realizava as tarefas da escola, entre outros problemas. Concomitantemente, os pais participaram do Treinamento de Pais. Em decorrência de desistências de outras crianças do grupo infantil, Lucas passou a ser atendido individualmente depois de completar cinco sessões grupais, e os pais passaram a participar do Treinamento de Pais individualmente, após duas sessões grupais com outros pais.

Como o presente estudo objetiva abordar as práticas parentais, cabe explicitar as práticas educacionais da mãe de Lucas. Esta relatou frequentes agressões físicas ao filho como chineladas e palmadas fortes; ela também apresentava falta de paciência com os comportamentos externalizantes do filho, negligência ao não estar ciente das necessidades afetivas, disciplina relaxada ao estabelecer ordens e não cumpri-las, e raras demonstrações de carinho. 0 pai justificava sua ausência na vida do filho pela carga horário de trabalho, contribuindo para a perpetuação da falta de carinho, falta de limites e negligência. No treinamento de pais foram abordadas questões sobre os conceitos de modelagem, reforço e punição, assim como a utilização de algumas técnicas com o filho, como maneiras de comunicação, o cantinho do pensamento, economia de fichas, orientações acerca da importância da interação por meio da brincadeira e do cumprimento dos limites dados, além de discussões sobre outras dificuldades.

Apesar de o Treinamento de Pais continuar ocorrendo com a mãe, esta não vinha aderindo ao que lhe era orientado, permanecia com falta de paciência para lidar com o filho e, consequentemente, as punições continuavam frequentes e os reforços dos comportamentos adequados escassos. A falta de aderência às práticas parentais positivas foi justificada pela mãe de Lucas pela sua falta de paciência, falta de tempo, muita irritação perante a conduta do filho, entre outros fatores que a estavam desmotivando. Diante dessas circunstâncias, Lucas não vinha apresentando progressos e a mãe desmotivou-se com o tratamento, não o trazendo mais aos atendimentos.

O segundo estudo de caso contempla a problemática de Adriana (35 anos), que chegou ao atendimento psicológico com queixas de não conseguir controlar as agressões psicológicas e físicas à filha de sete anos de idade, assim como agressão verbal a outras pessoas em contextos laborais e familiares. Ela expunha que a filha vinha apresentando problemas de comportamento como irritação, cumprimento das regras apenas por meio de gritos e de agressão física, retraimento, agressão à avó e a ela, além de dificuldades de expor-se em sala de aula.

Diante desses comportamentos da filha, Adriana vinha agredindo a menina, com palmadas frequentes, gritos, menosprezo, raros reforços, entre outras práticas coercitivas. Considerando esse contexto, concomitantemente ao trabalho de questões individuais, trabalhou-se com o Treinamento de Pais, tratando a importância da brincadeira com a filha e dos limites sem o uso da agressão, como dar ordens, o incentivo aos reforços, entre várias outras técnicas. Ressalta-se que a mãe de Adriana a agredia muito na 
infância. Hoje, Adriana vive com a mãe e com a filha, e o ex-marido vê a filha nos finais de semana.

Realizaram-se 27 sessões até a redação do presente estudo. Nesse período Adriana desenvolveu empatia pela sua filha, sentindo culpa pelas agressões e sofrimento após realizá-las. Deixou de praticar com a mesma frequência e intensidade as agressões físicas e verbais, aumentou o número de reforços, desenvolveu estratégias para lidar com a desobediência da filha e com a sua falta de paciência, entre tantas outras estratégias estabelecidas no dia a dia delas para evitar a presença de práticas coercitivas. Diante das novas práticas parentais mais saudáveis, Adriana observou uma melhora no diálogo com a filha, a diminuição dos gritos e aumento das explicações, aumento do respeito, maior participação da filha em aula segundo a avaliação da professora, diminuição da irritação da filha, maior cumprimento de regras estabelecidas com o uso apenas da ordem, além da diminuição das agressões físicas.

\section{Discussão}

Em ambos os casos observaram-se as dificuldades dos pais para aderir às práticas educacionais parentais positivas, mesmo quando estes tinham consciência de que a aderência a essas práticas trariam benefícios para a relação parental e para o desenvolvimento saudável dos filhos. A mãe de Lucas, apesar de referir ter consciência de que as práticas parentais coercitivas causavam problemas de comportamento no filho, não conseguiu exercer as práticas parentais positivas pela desmotivação ante a presença dos comportamentos externalizantes do filho, como pela falta de paciência e falta de tempo.

0 processo psicoterápico de Adriana também apresentou dificuldades de adesão a práticas parentais positivas, as quais estiveram relacionadas ao hábito de interação que ela sempre tivera ao longo dos anos com a filha, que contrastava com essa nova maneira de se comunicar com a sua filha e com outras pessoas. Observou-se que, gradualmente, ao longo das 27 sessões, a frequência e a intensidade das punições, agressões, menosprezos em relação à filha haviam diminuído e os reforços haviam aumentado. As dificuldades mencionadas acima são referidas por Silva, Del Prette A. e Del Prette Z. (2000), os quais afirmam que os pais que praticam práticas parentais coercitivas apresentam dificuldades no repertório da aplicação das práticas parentais positivas, como por exemplo, ao ter que deixar de praticar punições e expressar adequadamente os sentimentos negativos, mesmo esforçando-se para superar essas dificuldades conscientes.

Observou-se que um dos objetivos do Treinamento de Pais, dentre outros, foi conquistado por Adriana: o de reforçar positivamente os comportamentos que os pais querem ver com mais frequência nos filhos e estarem conscientes de que podem reforçar acidentalmente as crianças a fazer coisas que eles desaprovam (Pearl, 2009). Estar ciente desses reforços foi extremamente importante para que Adriana percebesse no dia a dia que essa relação realmente ocorria, e a partir disso modificar a sua maneira de interação com a filha. Um exemplo disso foi quando Adriana observou que, quanto mais ela gritava com a filha, mais a filha repetia o comportamento que Adriana queria extinguir. Em outras palavras, o comportamento indesejado da filha era a maneira que esta tinha de ganhar a atenção da mãe, que passava o dia todo trabalhando.

Salienta-se que Lucas e a família residiam em um ambiente caracterizado pela presença constante de policiais armados e usuários de drogas, os quais eram facilmente observados pelas crianças da comunidade; o ambiente também era marcado por conflitos entre as crianças da escola, que também, segundo o relato de Lucas, apresentavam problemas de comportamento externalizantes, e pela presença de práticas parentais coercitivas. Esses aspectos, como práticas parentais inadequadas, ambientes familiares adversos e rejeição de companheiros na escola, associam-se fortemente com os problemas de comportamento externalizantes das crianças (Szelbracikowski \& Dessen, 2007).

Destaca-se também, conforme o estudo de Costello, Compton e Keeler (2003), que crianças pertencentes a classes econômicas mais pobres tinham mais sintomas psiquiátricos (Transtorno de Conduta e Transtorno Desafiador de Oposição) do que crianças que nunca pertenceram a classes econômicas pobres. Logo, atentar para comunidades mais carentes economicamente torna-se relevante para intervenções destinadas à prevenção de problemas de comportamento externalizantes e futuro desenvolvimento de comportamentos antissociais. Ressalva-se essa observação, visto que no estudo de Copeland, MillerJohnson, Keeler, Angold e Costello (2007) crianças 
com padrões específicos de psicopatologia com e sem transtorno de conduta apresentaram risco de desenvolverem comportamentos criminosos mais tarde. 0 estudo conclui que identificar e tratar esses padrões disfuncionais diminui a criminalidade.

Outras variáveis sobre o uso de práticas parentais inadequadas seriam: estresse conjugal; problemas econômicos; perda de emprego; e padrões de funcionamento familiar. Conclui-se que o estresse é um dos fatores responsáveis pelo aumento da irritabilidade dos pais e pela probabilidade do uso de comportamentos agressivos com seus filhos (Szelbracikowski \& Dessen, 2007). A associação do estresse com as práticas coercitivas pode ser observada pelo relato de agressões inconsistentes de Adriana à sua filha; a mãe referia estar frequentemente estressada diante da preocupação financeira e da falta de tempo para realizar tudo que almejava.

Adriana sempre referiu em seus atendimentos que a mãe a educara de maneira muito rígida, por vezes com o uso de agressões físicas e verbais, e logo, a maneira que educava a filha estava muito relacionada ao que a mãe lhe ensinara. Assim, elogiar e reforçar a filha foram um dos maiores ganhos que Adriana referiu conquistar nesse processo de psicoterapia. Logo, torna-se importante compreender a transmissão de estilos e práticas parentais de pais para filhos para acabar com os padrões inadequados de comportamento (Weber, Selig, Bernardi \& Salvador, 2006), visto que há transmissão intergeracional da agressividade (Capaldi \& Clarck, 1998 citados por Weber et al., 2006, p. 409).

Os benefícios do Treinamento de Pais foram observados ao longo dos atendimentos no caso de Adriana, que, por mais dificuldades que tivesse, começou a utilizar práticas parentais mais positivas, o que repercutiu em comportamentos mais respeitosos da filha e na melhora do vínculo dessa díade. Adriana começou a brincar e a conversar com a filha, a controlar as agressões verbais e físicas, a reforçar os comportamentos desejáveis, a praticar o reforço negativo nos possíveis castigos, tomar ciência da modelação que influenciava os comportamentos da filha (assim como a mãe influenciara os dela).

Entretanto, não se observou o desenvolvimento mais saudável em Lucas, cujos pais, mesmo tendo outras questões que contribuíam para a presença dos comportamentos externalizantes, não ofereceram maior amparo para essas questões que propiciavam a presença de comportamentos indesejáveis.
Ressalva-se assim que os pais que desenvolvem práticas como discussões pacíficas e abordagens indutivas diante de confrontos incutem em seus filhos um senso de respeito perante visões diferentes e crenças de que disputas podem ser resolvidas por meios não aversivos (Mondin, 2008). Assim, torna-se relevante os pais estarem cientes e dispostos a vencerem os obstáculos que os impedem de aderirem a práticas parentais positivas para um desenvolvimento mais saudável de seus filhos.

\section{Considerações finais}

A presença de problemas de comportamento nos filhos está relacionada a múltiplas variáveis, dentre elas o ambiente, questões referentes ao diagnóstico, à modelagem dos pais e/ou de ídolos, problemas conjugais, estresse parental, entre outras. Diante de todas essas questões cabe aos pais tornarem-se cientes dos eventos que circundam os filhos e possibilitarem a estes um desenvolvimento saudável por meio de práticas parentais positivas, visto que quando estas são coercitivas repercutem de maneira negativa nos comportamentos das crianças.

Práticas parentais positivas podem ser vistas como importante fator de proteção no desenvolvimento posterior, possibilitando uma adolescência com menos problemas de comportamento externalizantes e consequentemente melhores prognósticos para essas crianças que sofrem danos importantes em suas características de personalidade. Sugere-se a realização de mais trabalhos neste sentido para contribuir para uma sociedade melhor e mais humanitária diante de indivíduos com menores chances de terem comportamentos antissociais.

\section{Referências}

Abib, J. A. D. (2001). Arqueologia do behaviorismo radical e o conceito de mente. In J. Guilhardi, et al. (Org.). Sobre comportamento e cognição - Expondo a variabilidade. (pp. 20-35). Santo André: Esetec.

Alvarenga, P., \& Piccinini, C. A. (2007). Preditores do desenvolvimento social na infância: Avanços, a seleção por conseqüências em ação. (pp. 536-541). Santo André: Esetec. 
Alvarenga, P., \& Piccinini C. A. (2009). Práticas educativas maternas e indicadores do desenvolvimento social no terceiro ano de vida. Psicologia Reflexão e Crítica, 22(2).

Bandeira, M., Rocha, S. S., Souza, T. M. P., Del Prette, Z. A. P., \& Del Prette, A. (2006). Comportamentos problemáticos em estudantes do ensino fundamental: Características da ocorrência e relação com habilidades sociais e dificuldades de aprendizagem. Estudos em Psicologia, 11(2), 199-208.

Bandura, A. (1973). Aggression: A social learning analysis. Englewood Cliffs, NJ: Prentice-Hall.

Bolsoni Silva, A. T., \& Marturano, E. M. (2002). Práticas educativas e problemas de comportamento: Uma análise à luz das habilidades sociais. Estudos de Psicologia, 7(2), 227-235.

Bolsoni-Silva, A. B., Del Prette, A., \& Oishi, J. (2003). Habilidades sociais de pais e problemas de comportamento nos filhos. Psicologia Argumento, 9, 11-29.

Coelho, M. V., \& Murta, S. G. (2007). Treinamento de pais em grupo: um relato de experiência comportamento e cognição. Estudos de Psicologia, 24(3).

Conte, F. C. S. (2001). Promovendo a relação entre pais e filhos. In M. Delitti (Org.). Sobre comportamento $e$ cognição: A prática da análise do comportamento e da terapia cognitivo-comportamental. (pp 161-168). Santo André: Esetec.

Copeland, W. E., Miller-Johnson, S., Keeler, G., Angold, A., \& Costello, E. J. (2007). Childhood psychiatric disorders and young adult crime: a prospective, populationbased study. Am J Psychiatry, 164, 1668-1675.

Cortez, M. B., Padovani R. C., \& Williams, L. C. A. (2005). Terapia de grupo cognitivo-comportamental com agressores conjugais. Estudos de Psicologia, 22(1).

Costello, J. E., Compton, S. N., \& Keeler, G. (2003). Relationships between poverty and psychopathology: A natural experiment. JAMA, 290(15), 2023-2029.

Del Prette, A., \& Del Prette, Z. A. P. (2002). Psicologia das relações interpessoais: Vivências para o trabalho em grupo. Petrópolis: Vozes.

Gallo, A. E., \& Williams, L. C. A. (2005). Adolescentes em conflito com a lei: Uma revisão dos fatores de risco para a conduta infracional. Psicologia: Teoria $e$ Prática, 7(1), 81-95.
Gomide, P. I. C. (2001). Efeitos das práticas educativas no desenvolvimento do comportamento anti-social. In M. L. Marinho; V. E. Caballo (Org.). Psicologia clínica e da saúde. (pp. 33-39). Londrina: UEL; Granada: Apicsa.

Kristensen, C. H., Lima J. S., Ferlin, M., Flores, R. Z., \& Hackmann, P. H. (2003). Fatores etiológicos da agressão física: Uma revisão teórica. Estudos de Psicologia, 8(1).

Lochman, J. E., Powell, N. R., Whidby, J. M., \& Fitzgerald, D. P. (2006). Cognitive-Behavioral Assessment and Treatment with Aggressive Children. In P. C. Kendall (Ed.). Child and adolescent therapy: Cognitive Behavioral producers. (pp. 33-81). New York: Guilford Press.

Lubi, A. P. L. (2003). Estilo parental e comportamento socialmente habilidoso da criança com pares. In Brandão, M. Z., Conte, F. C. S., Brandão F. S. Ingberman, Y. K., Moura, C. B., Silva, V. M., et al (Org.). Sobre Comportamento e Cognição: A história, os avanços, a seleção por conseqüências em ação. (Vol. 11, pp. 536541). Santo André: Esetec.

Marlowe, B. A., \& Canestrari, A. S. (2006). Transmission of aggression through imitation of aggressive models. In B. A. Marlowe \& A. S. Canestrari. Educational psychology in context. Readings for Future Teachers. (2. ed.). Sage Publications.

Mondin, E. M. C. (2008). Práticas educativas parentais e seus efeitos na criação dos filhos. Psicologia Argumento, 26(54), 233-244.

Pearl, E. S. (2009). Parent management training for reducing oppositional and aggressive behavior in preschoolers. Aggression and Violent Behavior, 14, 295-305.

Pesce, R. (2009). Violência familiar e comportamento agressivo e transgressor na infância: Uma revisão da literatura. Ciência saúde coletiva, 14(2).

Piccinini, C. A., Frizzo G. B., Alvarenga P., Lopes R. S., \& Tudge J. (2007). Práticas educativas de pais e mães de crianças aos 18 meses de idade. Psicologia: Teoria e Pesquisa, 23(4).

Salvador, A. P. V., \& Weber, L. N. D. (2005). Práticas educativas parentais: Um estudo comparativo da interação familiar de dois adolescentes distintos. Interação em Psicologia, 9(2), 341-353.

Salvo, C. G., Silvares, E. F. M., \& Toni, P.M. (2005). Práticas educativas como forma de predição de problemas de comportamento e competência social. Estudos de Psicologia Campinas, 22(2),187-195. 
Sapienza, G., Aznar-Farias, M., \& Silvares, E. F. M. (2009). Competência social e práticas educativas parentais em adolescentes com alto e baixo rendimento acadêmico. Psicologia Reflexão e Crítica, 22(2).

Serra-Pinheiro, M. A., Guimarães, M. M., \& Serrano, M. E. (2005). A eficácia de treinamento de pais em grupo para pacientes com transtorno desafiador de oposição: Um estudo piloto. Revista Psiquiatria Clínica, 32(2),68-72.

Silva, A. T. B., Del Prette, A., \& Del Prette, Z. A. P. (2000). Relacionamento pais filhos: um programa de desenvolvimento interpessoal em grupo. Psicologia escolar e educacional, 3(3), 203-215.

Szelbracikowski, A. C., \& Dessen, M. A. (2007). Problemas de comportamento exteriorizado e as relações familiares: Revisão de literatura. Psicologia em Estudo, 12(1), 33-40.
Todorov, J. C., \& Moreira, M. B. (2009). Psicologia, comportamento, processos e interações. Psicologia, Reflexão e Crítica. 22(3),404-412.

Velásquez, R., Souza, S. D., Adjuto, I., Muñoz, L. M., \& Silveira, J. C. C. (2010). 0 treinamento de pais e cuidadores: ensinando a educar e promovendo a saúde mental. Revista Médica Minas Gerais, 20(2), 182-188.

Weber, L. N. D., Selig, G. A, Bernardi, M. G., \& Salvador, A. P. V. (2006). Continuidade dos estilos parentais através das gerações - transmissão intergeracional de estilos parentais. Paidéia,16(35).

Ventura, M. M. (2007). O estudo de caso como modalidade de pesquisa. Revista Socerj, 20(5), 383-386.

Yin, R. (2005). Estudo de Caso: Planejamento e métodos. São Paulo: Bookman. 